\title{
Sulfapyridine Sodium
}

National Cancer Institute

\section{Source}

National Cancer Institute. Sulfapyridine Sodium. NCI Thesaurus. Code C73842.

A sodium salft form of sulfapyridine, a sulfonamide antibiotic and by product of sulfasalazine. 